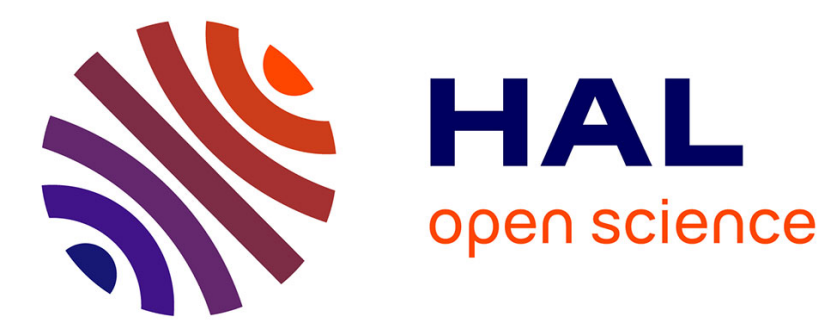

\title{
L'enrôlement du féminisme dans la lutte contre la prostitution
}

\author{
Lilian Mathieu
}

\section{To cite this version:}

Lilian Mathieu. L'enrôlement du féminisme dans la lutte contre la prostitution. Cités: Philosophie, politique, Histoire, 2018, 73, pp.57-66. halshs-02057159

\section{HAL Id: halshs-02057159 \\ https://shs.hal.science/halshs-02057159}

Submitted on 5 Mar 2019

HAL is a multi-disciplinary open access archive for the deposit and dissemination of scientific research documents, whether they are published or not. The documents may come from teaching and research institutions in France or abroad, or from public or private research centers.
L'archive ouverte pluridisciplinaire HAL, est destinée au dépôt et à la diffusion de documents scientifiques de niveau recherche, publiés ou non, émanant des établissements d'enseignement et de recherche français ou étrangers, des laboratoires publics ou privés. 


\section{L'enrôlement du féminisme dans la lutte contre la prostitution}

\section{Lilian Mathieu}

Lorsqu'en juin 1975 les féministes françaises se rallièrent à la cause des prostituées, elles le firent au nom d'une solidarité féminine contre la répression menée par cette institution patriarcale qu'est la police ${ }^{1}$. Ce n'est pas sans une certaine ironie qu'on a pu voir, une quarantaine d'années plus tard, de larges pans du mouvement des femmes applaudir au vote d'une loi qui confie à la police l'essentiel d'une politique destinée à abolir la prostitution. De fait, ces fractions, majoritaires au sein de l'espace de la cause des femmes ${ }^{2}$, n'ont pas fait qu'applaudir l'adoption de la loi du 13 avril 2016 «renforçant la lutte contre le système prostitutionnel », dont la mesure phare est la pénalisation des clients des prostituées. Elles l'ont préparée, soutenue et légitimée au cours d'un long travail de lobbying dont la dernière étape fut la campagne «Abolition 2012 » à laquelle participèrent des structures féministes telles qu'Osez le féminisme, Choisir la cause des femmes, SOS sexisme, Femmes solidaires, Encore féministes, les Chiennes de garde, le Lobby européen des femmes, le Collectif droits des femmes, la Coordination lesbienne de France ou encore la Marche mondiale des femmes.

Là n'est pas la seule ironie. En 1975, la mobilisation des prostituées avait bénéficié de l'impulsion et du soutien décisifs d'une association abolitionniste, le Mouvement du Nid. Son identité catholique avait suscité la défiance de féministes dont le combat pour la légalisation de l'avortement venait à peine de triompher. Quatre décennies ans plus tard, c'est en étroite association avec ce même Mouvement du Nid que les féministes ralliées à l'abolitionnisme ont mené campagne pour la pénalisation des clients. Certes, c'était de manière impromptue que les féministes avaient en 1975 soutenu les prostituées. La prostitution n'avait jusqu'alors guère été débattue au sein du mouvement des femmes et ce n'est qu'a posteriori qu'une réflexion s'était ébauchée en dessinant les linéaments d'une critique de la vente institutionnalisée de «services sexuels» par des femmes et pour des hommes ${ }^{3}$. Mais les arguments intellectuels n'ont pas été seuls à l'œuvre dans la conversion d'une part majoritaire du féminisme français au projet d'une abolition de la prostitution. C'est davantage sous

\footnotetext{
${ }^{1}$ Un dessin à la une de Libération du 11 juin 1975 représente un CRS frappant une femme à terre avec une matraque de forme phallique. Sur cet épisode, voir Lilian Mathieu, Mobilisations de prostituées, Paris, Belin, 2001.

${ }^{2}$ On emprunte cette notion à Laure Bereni, La bataille de la parité, Paris, Economica, 2015.

${ }^{3}$ Voir par exemple Annie Mignard, «Propos élémentaires sur la prostitution », Les Temps modernes, $\mathrm{n}^{\circ} 356$, 1976.
} 
l'angle d'évolutions et de recompositions à la fois militantes et institutionnelles que doivent être envisagées les conditions de leur enrôlement dans ce combat ${ }^{4}$.

\section{Une coalition improbable}

Abolitionnisme d'inspiration catholique et féminisme ont longtemps entretenu des relations distantes et marquées par la défiance. Le Mouvement du Nid défendait encore dans les années 1990 des positions conservatrices en matière de mœurs et de sexualité, déplorant par exemple la banalisation du divorce et donnant la parole au prêtre et psychanalyste Tony Anatrella pour dénoncer les unions homosexuelles et s'en prendre à une libération sexuelle aux effets selon lui délétères ${ }^{5}$. Son affiliation religieuse ne pouvait que susciter les réticences d'un mouvement des femmes dont la remobilisation du milieu des années 1990 s'est précisément opérée pour protéger un droit à l'avortement alors menacé par une opposition soutenue par l'Église catholique. Il a fallu, pour que le féminisme ravivé par la défense des droits reproductifs se rallie à l'abolitionnisme, que ce que la sociologie des mouvements

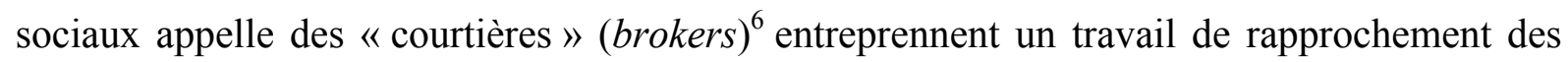
deux mouvances, prélude à leur coalition. Quatre personnalités ont joué un tel rôle.

La première est la journaliste Florence Montreynaud, ancienne militante du Planning familial et de Choisir mais surtout connue comme fondatrice des Chiennes de garde, une association qui dénonce le sexisme dans la publicité et dans la vie politique. Elle publie en 1993 un livre iconographique consacré à la prostitution, dans lequel elle interroge le regard du client sur cette activité ${ }^{7}$. Cet ouvrage, salué par le Mouvement du Nid, est non seulement à l'origine d'une attention plus vive de son auteure pour les clients mais également de son rapprochement avec l'abolitionnisme. Elle tient à la fin des années 1990 une rubrique régulière dans Prostitution et société intitulée « Payer pour ça », dans laquelle elle se livre à une dénonciation sarcastique de cette pratique spécifiquement masculine qu'est l'achat de « services sexuels ».

La deuxième courtière est la sociologue Marie-Victoire Louis. Antérieurement surtout spécialisée en sociologie du travail et active dans la lutte contre le harcèlement sexuel, elle commence à s'intéresser à la prostitution au début des années 1990, tout d'abord comme observatrice des débats sur le sujet au sein des institutions européennes. Elle se fait à ce titre

\footnotetext{
${ }^{4}$ Cet article reprend et actualise des éléments présentés dans Lilian Mathieu, La Fin du tapin. Sociologie de la croisade pour l'abolition de la prostitution, Paris, F. Bourin, 2014.

${ }^{5}$ Voir les n ${ }^{\circ} 92$ (1991) et 102 (1993) de la revue du Mouvement, Prostitution et société.

${ }^{6}$ Doug McAdam, Sidney Tarrow, Charles Tilly, Dynamics of Contention, Cambridge, Cambridge University Press, 2001.

${ }^{7}$ Florence Montreynaud, Amours à vendre, Paris, Glénat, 1993.
} 
la virulente dénonciatrice de la politique de la Hollande — qui normalise à partir de 2000 l'exercice de la prostitution et dépénalise certaines activités relevant antérieurement du proxénétisme - et se pose en lanceuse d'alerte devant ce qu'elle perçoit comme une entreprise de conversion d'autres pays à la reconnaissance du «travail du sexe». Son statut académique la légitime rapidement comme experte de la prostitution, régulièrement invitée à s'exprimer lors des colloques et dans la presse des associations abolitionnistes.

La troisième courtière est Malka Marcovich, représentante du Mouvement pour l'abolition de la prostitution et de la pornographie (MAPP) qui est l'antenne française de la « Coalition against trafficking in women » (CATW), organisation issue du féminisme radical américain. Marcovich est en 2002 l'auteure d'un rapport pour la Commission nationale contre les violences envers les femmes intitulé Le système de la prostitution: une violence à l'encontre des femmes, amplement relayé au sein des groupes féministes et dans lequel elle décrit « l'acte sexuel marchand comme la clé de voûte de la persistance des inégalités entre hommes et femmes $»^{8}$.

Ces trois personnalités s'identifient explicitement comme féministes et leurs prises de positions connaissent une rapide et large diffusion au sein de l'espace de la cause des femmes. Principale structure issue de la remobilisation féministe du milieu des années 1990, le Collectif national pour les droits des femmes (CNDF) s'approprie l'expression de « système prostitutionnel» forgée par Louis ${ }^{9}$ tandis que Montreynaud et Marcovich sont invitées en 2002 à son forum « De nouveaux défis pour le féminisme ». Elles y interviennent à la même tribune que la journaliste du Nid Claudine Legardinier qui, elle aussi mais cette fois du côté abolitionniste, travaille au rapprochement entre les deux mouvances. C'est elle qui sera la principale artisane de l'identification féministe du Mouvement du Nid, au point d'affirmer dans sa revue que «le caractère central du féminisme dans le choix de la position abolitionniste saute aux yeux. Son analyse du système de domination sexiste comme structure fondamentale de nos sociétés lui permet de voir la prostitution comme un point d'exaspération de la domination masculine, de la violence et de l'inégalité entre les hommes et les femmes $»^{10}$.

\footnotetext{
${ }^{8}$ Malka Marcovich, Le système de la prostitution : une violence à l'encontre des femmes, Commission nationale contre les violences envers les femmes, 2002, non paginé.

${ }^{9}$ La prostitution est pour elle un « système de domination marchande sur les sexes, sur les corps et donc sur les êtres humains qui, par la force, la contrainte et la violence légitimées par le droit, crée les conditions d'une mise en relation de "clients" auxquels des proxénètes - personnes physiques et morales - garantissent, contre rémunération, la possibilité d'un accès marchand aux sexes d'autres personnes, de sexe féminin dans la grande majorité des cas, femmes adultes, adolescentes, petites filles »; Marie Victoire Louis, « Prostitution », in Gérard Lopez, Stamatios Tzitzis (dir.), Dictionnaire de sciences criminelles, Paris, Dalloz, p. 759.

${ }^{10}$ Prostitution et société, $\mathrm{n}^{\circ} 166,2009$.
} 
Moment important, le forum du CNDF accueille également Günila Ekkberg, fémocrate ${ }^{11}$ suédoise alors chargée par le gouvernement de son pays d'assurer la promotion internationale de sa loi, adoptée en 1999, pénalisant l'achat de prestations prostitutionnelles. La Suède a en effet été le premier pays à considérer les clients des prostituées comme des délinquants passibles d'une lourde amende et de six mois de prison. Cette disposition pénale a été défendue par des parlementaires féministes et a pris place parmi un ensemble de mesures destinées à lutter contre les violences sexistes. Définir la prostitution comme une violence dont les principaux auteurs, les hommes clients, doivent par conséquent être sanctionnés ne pouvait que rencontrer un vif intérêt au sein d'un mouvement des femmes français qui s'est historiquement constitué dans le combat contre les violences faites aux femmes ${ }^{12}$.

\section{Les relais institutionnels du féminisme}

C'est à la fin des années 1990, sous l'impulsion première des associations abolitionnistes bientôt rejointes par de larges pans du mouvement des femmes, que se dessine une mobilisation revendiquant l'objectif d'une abolition de la prostitution. Celle-ci bénéficie d'un contexte politique et institutionnel favorable, sous l'effet de ce que Laure Bereni et Anne Revillard ont décrit comme une acclimatation institutionnelle du féminisme ${ }^{13}$. Après avoir été longuement dénoncée, la marginalisation politique des femmes fait l'objet de réponses institutionnelles : création en 1995 de l'Observatoire de la parité, nomination en 1997 d'une déléguée interministérielle aux Droits des femmes puis, en 1999, adoption de la première loi sur la parité. La même année voit la création de délégations parlementaires aux Droits des femmes, saisies dès qu'un projet ou une proposition de loi concerne l'égalité hommesfemmes. Celle du Sénat, présidée par la socialiste Dinah Dericke, consacre une partie importante de son rapport d'activité de 1999 à la prostitution ${ }^{14}$.

Ce rapport, pour la préparation duquel personnalités et organisations tant féministes qu'abolitionnistes (M.-V. Louis, Mouvement du Nid, MAPP, Coordination française du Lobby européen des femmes, etc.) ont été auditionnées, est le premier d'une longue série de

\footnotetext{
${ }^{11}$ Ce terme, qui désigne celles qui font avancer la cause des femmes de l'intérieur des institutions, a été forgé par Lee Ann Banaszak, The Women' Movement Inside and Outside the State, Cambridge, Cambridge University Press, 2010.

${ }^{12}$ Pauline Delage, Violences conjugales. Du combat féministe à la cause publique, Paris, Presses de Sciences-Po, 2017.

${ }^{13}$ Laure Bereni, Anne Revillard, « Des quotas à la parité : “féminisme d'État” et représentation politique (19742007) », Genèses, n 67, 2007.

${ }^{14}$ Dinah Dericke, Les Politiques publiques et la prostitution, Rapport d'information sur l'activité de la délégation aux droits des femmes et à l'égalité des chances entre les hommes et les femmes pour l'année 2000, Sénat, $n^{\circ} 209$, 2000-2001.
} 
travaux parlementaires sur le sujet ${ }^{15}$. En 2001, la mission d'information sur l'esclavage moderne auditionne à son tour le Mouvement du Nid et le MAPP sur la question de la traite des êtres humains ${ }^{16}$. Un an plus tard, la présidente socialiste de la Commission nationale contre les violences envers les femmes confie à M. Marcovich la rédaction de son rapport sur le «système de la prostitution ». La préparation des articles relatifs à la prostitution de la Loi sur la sécurité intérieure de mars 2003 donne elle aussi lieu à des rapports parlementaires, qui rappellent combien l'existence de la prostitution est liée à celle des inégalités entre hommes et femmes ${ }^{17}$. Suivront le rapport de la mission d'information sur la prostitution préparé par les députés Danielle Bousquet et Guy Geoffroy ${ }^{18}$ (qui, le premier, préconise de pénaliser les clients des prostituées), celui des sénateurs Chantal Jouanno et Jean-Pierre Godefroy sur la situation sanitaire et sociale des prostituées ${ }^{19}$ ainsi que ceux, produits tant au Sénat qu'à l'Assemblée nationale, qui accompagnent la discussion de la proposition de loi renforçant la lutte contre le système prostitutionnel, adoptée le 13 avril 2016. Celle-ci avait été précédée par le vote par l'Assemblée nationale, en décembre 2011, d'une résolution « réaffirmant la position abolitionniste de la France en matière de prostitution » et par la loi du 4 août 2014 pour l'égalité réelle entre les femmes et les hommes dont l'article $1^{\mathrm{er}}$ stipule que la politique pour l'égalité entre les femmes et les hommes comporte notamment des actions visant à renforcer la lutte contre le «système prostitutionnel ».

Tout, bien sûr, ne s'est pas joué dans les arènes institutionnelles. On l'a dit, la campagne «Abolition 2012 » qui a assuré la promotion de la pénalisation des clients a vu l'implication d'un large éventail d'associations à l'identité féministe revendiquée. Organisation de rencontres-débats dans toute la France, tribunes de personnalités dans la presse, prises de positions publiques de porte-parole associatives, rassemblements et manifestations, etc. ont donné une ample visibilité médiatique à la campagne et conféré un poids certain à ses interpellations du champ politique.

\footnotetext{
${ }^{15}$ Dinah Dericke, Les Politiques publiques et la prostitution. Rapport d'information sur l'activité de la délégation aux droits des femmes et à l'égalité des chances entre les hommes et les femmes pour l'année 2000, Sénat, $\mathrm{n}^{\circ} 209,2000-2001$.

${ }^{16}$ Christine Lazerges, Alain Vidalies, Rapport de la mission d'information commune sur les diverses formes de l'esclavage moderne, Assemblée nationale, $\mathrm{n}^{\circ}$ 3459, 2001.

${ }^{17}$ Janine Rozier, Rapport d'information fait au nom de la Délégation au droit des femmes et à l'égalité des chances entre les hommes et les femmes sur le projet de loi $n^{\circ} 30$ (2002-2003) pour la sécurité intérieure, Sénat, $\mathrm{n}^{\circ}$ 34, 29 octobre 2002, et Marie-Jo Zimmermann, Rapport d'information fait au nom de la Délégation au droit des femmes et à l'égalité des chances entre les hommes et les femmes sur le projet de loi adopté par le Sénat après déclaration d'urgence ( $\left.n^{\circ} 381\right)$ pour la sécurité intérieure, Assemblée nationale, $n^{\circ} 459,5$ décembre 2002. ${ }^{18}$ Danielle Bousquet, Guy Geoffroy, Prostitution : l'exigence de responsabilité. En finir avec le mythe du "plus vieux métier du monde », Assemblée nationale, Commission des lois, $\mathrm{n}^{\circ}$ 3334, 2011.

${ }^{19}$ Chantal Jouanno et Jean-Pierre Godefroy, Situation sanitaire et sociale des personnes prostituées : inverser le regard, Sénat, Commission des affaires sociales, $n^{\circ} 46,8$ octobre 2013.
} 
La représentation d'une revendication portée par un mouvement social et adoptée sous sa pression par des responsables politiques serait pourtant trompeuse. Solidaire d'une conception des mouvements sociaux comme nécessairement extérieurs à un champ politique étanche, elle méconnaît que les institutions comptent fréquemment en leur sein des militants (ou ex-militants) entendant y défendre les valeurs et projets qui leur sont chers. C'est spécialement le cas du féminisme dont l'institutionnalisation au sein de l'État a offert des opportunités de carrière à ses militantes aux qualifications les mieux ajustées — et que le terme de fémocrates vise donc à désigner. C'est spécialement le cas ici, puisque la gestion administrative du traitement social de la prostitution (dont les associations abolitionnistes tirent une large part de leurs financements) a été confiée à la fin des années 1990 au Service des Droits des femmes au sein de la Direction générale des affaires sociales ${ }^{20}$. La cooptation institutionnelle de représentants associatifs favorise pour sa part une homogénéisation des représentations et des positions d'acteurs multipositionnés dans et hors de l'État: C. Legardinier n'est ainsi pas seulement journaliste à Prostitution et société mais a été membre de l'Observatoire de la parité entre 2002 et 2005, tandis que le secrétaire général du Mouvement du Nid, Grégoire Théry, est membre du Haut conseil à l'égalité entre les hommes et les femmes qui lui a succédé - un Conseil présidé par l'ancienne députée Danièle Bousquet, co-auteure du rapport parlementaire sur la prostitution de 2011. On remarquera également que les organisations féministes les plus investies dans la campagne «Abolition $2012 »$ sont souvent les plus institutionnalisées et les plus proches des pouvoirs publics ${ }^{21}$. La pénalisation des clients doit ainsi être envisagée comme une coproduction du champ politique et des mouvements abolitionniste et féministe, facilitée par l'institutionnalisation désormais bien avancée de ce dernier.

\section{Les limites d'un féminisme univoque}

Ce n'est bien sûr pas l'ensemble du mouvement des femmes qui s'est rallié à la revendication d'une abolition de la prostitution dont la pénalisation des clients serait l'instrument principal. De nombreuses personnalités et organisations se revendiquant du féminisme (dont, en premier lieu, le Mouvement français pour le planning familial) ont exprimé leur désaccord avec une mesure qui ne peut selon elles que renforcer la précarité et la clandestinité des prostituées. D’autres, appartenant surtout aux secteurs de la santé publique

\footnotetext{
${ }^{20}$ Anne Revillard, La Cause des femmes dans l'État, Grenoble, PUG, 2016.

${ }^{21}$ Ibid., p. 229. C'est notamment le cas du Lobby européen des femmes, dont l'essentiel des financements provient de la Commission européenne et qui exerce un quasi monopole sur la représentation des intérêts des femmes au niveau européen.
} 
(tels Médecins du monde) et du mouvement homosexuel (à l'instar d'Act Up), ont également pointé combien une telle mesure apparaissait régressive en regard des acquis de trente ans de lutte contre le VIH-sida, lesquels ont largement attesté la contre-productivité des options coercitives $^{22}$. De même, une lecture unilatéralement féministe conduit-elle à une appréhension réductrice de la prostitution en occultant celle exercée par des hommes et des trans, dont les conditions d'exercice ne sont pas moins tramées par des rapports de domination que celles des femmes ${ }^{23}$. Enfin, la définition univoque des prostituées comme victimes passives de l'oppression patriarcale pourrait bien être l'expression d'un misérabilisme où se mêlent condescendances de genre, de classe et de « race ».

Les controverses, souvent vives, suscitées par le thème de la prostitution au sein $d u$ féminisme français paraissent reproduire, avec quelques décennies de distance, les fameuses sex wars qui, sur la question proche de la pornographie, avaient clivé le mouvement des femmes américain à la fin des années $1970^{24}$. S'y étaient déjà exprimés le refus de certains courants de n'envisager la sexualité féminine qu'en termes d'oppression masculine et la crainte qu'une telle conception ne rejoigne le puritanisme réactionnaire de la droite religieuse $^{25}$. Le Mouvement du Nid est certes issu du catholicisme social et ce sont avant tout des partis de gauche qui ont défendu la loi d'avril 2016 alors que la droite réclamait le maintien de la pénalisation du racolage. Reste que la nouvelle législation n'appréhende pas tant la prostitution comme la résultante de structures économiques, sociales et genrées inégalitaires que comme le produit de l'action purement individuelle de figures délinquantes (proxénètes et clients), et entérine le passage d'une conception redistributrice de la justice (dont l'emblème est le travailleur social) vers une vision punitive (incarnée par policier) de celle-ci. Le soutien accordé par de larges pans du mouvement des femmes français à cette politique pénale se rapproche ce faisant de ce que Elizabeth Bernstein désigne aux États-Unis comme un «féminisme carcéral», désormais converti à la transfiguration néolibérale des problèmes sociaux en problèmes de sécurité ${ }^{26}$.

\footnotetext{
${ }^{22}$ Cela dans les domaines de la prostitution comme de la toxicomanie. Voir sur ce point le bilan récent de François Berdougo et Gabriel Girard, La Fin du sida est-elle possible ?, Paris, Textuel, 2017.

${ }^{23}$ Sur les proximités qui unissent prostitutions masculines et féminines, voir Lilian Mathieu, Sociologie de la prostitution, Paris, La Découverte.

${ }^{24}$ Clivage dont témoigne notamment l'ouvrage Pleasure and Danger. Exploring Female Sexuality dirigé par Carol Vance (Boston, Routledge \& Kegan Paul, 1984).

${ }^{25}$ Jonction qu'opérèrent les féministes radicales Catharine MacKinnon et Andrea Dworkin pour soutenir leur projet d'interdiction de la pornographie.

${ }^{26}$ Elizabeth Bernstein, « Militarized Humanitarianism Meets Carceral Feminism: The Politics of Sex, Rights, and Freedom in Contemporary Antitrafficking Campaigns », Signs, 36 (1), 2010.
} 